\title{
Some weighted Simpson type inequalities for differentiable s-convex functions and their applications
}

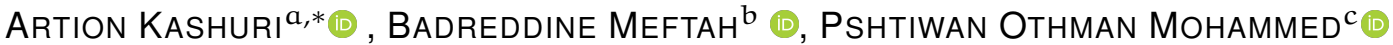 \\ a Department of Mathematics, Faculty of Technical Science, University "Ismail Qemali", \\ 9400, Vlora, Albania \\ b Laboratoire des télécommunications, Faculté des Sciences et de la Technologie, University \\ of 8 May 1945 Guelma, P.O. Box 401, 24000 Guelma, Algeria \\ ${ }^{c}$ Department of Mathematics, College of Education, University of Sulaimani, Sulaimani, \\ Kurdistan Region, Iraq
}

\author{
- Received: 20.02.2021 • Accepted: 05.03.2021 • Published Online: 06.03.2021
}

\begin{abstract}
In this study, by using a new identity we establish some new Simpson type inequalities for differentiable s-convex functions in the second sense. Various special cases have been studied in details. Also, in order to illustrate the efficient of our main results, some applications to special means and weighted Simpson quadrature formula are given. The obtained results generalize and refine certain known results. At the end, a brief conclusion is given as well.
\end{abstract}

Keywords: Simpson inequality, weighted function, s-convex functions, Hölder inequality, weighted Simpson quadrature formula.

2010 MSC: Primary: 26A51; Secondary: 05A30, 26A33, 26D07, 26D10, 26 D15.

\section{Introduction}

Definition 1.1. ([1]) Let $I$ be an interval of real numbers. A function $\psi: I \rightarrow R$ is said to be convex, if for all $\lambda_{1}, \lambda_{2} \in \mathrm{I}$ and all $\chi \in[0,1]$, we have

$$
\psi\left(\chi \lambda_{1}+(1-\chi) \lambda_{2}\right) \leqslant \chi \psi\left(\lambda_{1}\right)+(1-\chi) \psi\left(\lambda_{2}\right) .
$$

The concept of convex functions has been also generalized in diverse manners. One of them is the so-called s-convex function defined as follows:

\footnotetext{
*Corresponding author: artionkashuri@gmail.com 
Definition 1.2. ([2]) A nonnegative function $\psi: I \subset[0, \infty) \rightarrow R$ is said to be s-convex in the second sense for some fixed $s \in(0,1]$, if

$$
\psi\left(\chi \lambda_{1}+(1-\chi) \lambda_{2}\right) \leqslant \chi^{s} \psi\left(\lambda_{1}\right)+(1-\chi)^{s} \psi\left(\lambda_{2}\right)
$$

holds for all $\lambda_{1}, \lambda_{2} \in \mathrm{I}$ and $\chi \in[0,1]$.

The following inequality is known in the literature as Simpson's inequality.

Theorem 1.3. Let $\psi$ be four times continuously differentiable function on $\left(\lambda_{1}, \lambda_{2}\right)$ and $\left\|\psi^{(4)}\right\|_{\infty}$ $:=\sup _{x \in\left(\lambda_{1}, \lambda_{2}\right)}\left|\psi^{(4)}(x)\right|<\infty$, then

$$
\left|\frac{1}{6}\left(\psi\left(\lambda_{1}\right)+4 \psi\left(\frac{\lambda_{1}+\lambda_{2}}{2}\right)+\psi\left(\lambda_{2}\right)\right)-\frac{1}{\lambda_{2}-\lambda_{1}} \int_{\lambda_{1}}^{\lambda_{2}} \psi(u) d u\right| \leqslant \frac{1}{2880}\left\|\psi^{(4)}\right\|_{\infty}\left(\lambda_{2}-\lambda_{1}\right)^{4} .
$$

In recent years, many researchers have studied the error estimates of Simpson's inequality, in order to establish new refinements, generalizations as well as new Simpsontype inequalities for more details we refer readers $[3,4,5,6,7,8,9]$. For an overview, the reader should see also the following literatures on integral inequalities, $[14,15,16$, $17,18,19,20,21,22,23,24,25,26,27,28,29,30]$ and the references therein. The following notations will be used in sequel. We denote, respectively $\mathrm{I}^{\circ}$ the interior of $\mathrm{I}$ and $\mathcal{L}\left[\lambda_{1}, \lambda_{2}\right]$ the set of all integrable functions on $\left[\lambda_{1}, \lambda_{2}\right]$. In [10], Alomari et al. established the following Simpson type inequalities for s-convex functions.

Theorem 1.4. Let $\psi: \mathrm{I} \subset[0, \infty) \rightarrow \mathrm{R}$ be a differentiable mapping on $\mathrm{I}^{\circ}$ such that $\psi^{\prime} \in$ $\mathcal{L}\left[\lambda_{1}, \lambda_{2}\right]$, where $\lambda_{1}, \lambda_{2} \in \mathrm{I}^{\circ}$ with $\lambda_{1}<\lambda_{2}$. If $\left|\psi^{\prime}\right|$ is s-convex on $\left[\lambda_{1}, \lambda_{2}\right]$ for some fixed $s \in(0,1]$, then the following inequality holds:

$$
\begin{aligned}
& \left|\frac{1}{6}\left(\psi\left(\lambda_{1}\right)+4 \psi\left(\frac{\lambda_{1}+\lambda_{2}}{2}\right)+\psi\left(\lambda_{2}\right)\right)-\frac{1}{\lambda_{2}-\lambda_{1}} \int_{\lambda_{1}}^{\lambda_{2}} \psi(u) d u\right| \\
& \leqslant\left(\lambda_{2}-\lambda_{1}\right) \frac{6^{-s}-9 \times 2^{-s}+5^{s+2} \times 6^{-s}+3 s-12}{18\left(s^{2}+3 s+2\right)}\left[\left|\psi^{\prime}\left(\lambda_{1}\right)\right|+\left|\psi^{\prime}\left(\lambda_{2}\right)\right|\right] .
\end{aligned}
$$

Theorem 1.5. Let $\psi: \mathrm{I} \subset[0, \infty) \rightarrow \mathrm{R}$ be a differentiable mapping on $\mathrm{I}^{\circ}$ such that $\psi^{\prime} \in$ $\mathcal{L}\left[\lambda_{1}, \lambda_{2}\right]$, where $\lambda_{1}, \lambda_{2} \in I^{\circ}$ with $\lambda_{1}<\lambda_{2}$. If $\left|\psi^{\prime}\right|^{\mathrm{p} /(\mathrm{p}-1)}$ is s-convex on $\left[\lambda_{1}, \lambda_{2}\right]$ for some fixed 
$s \in(0,1]$ and $p \geqslant 1$, then the following inequality holds:

$$
\begin{aligned}
& \left|\frac{1}{6}\left(\psi\left(\lambda_{1}\right)+4 \psi\left(\frac{\lambda_{1}+\lambda_{2}}{2}\right)+\psi\left(\lambda_{2}\right)\right)-\frac{1}{\lambda_{2}-\lambda_{1}} \int_{\lambda_{1}}^{\lambda_{2}} \psi(u) d u\right| \\
\leqslant & \frac{\left(\lambda_{2}-\lambda_{1}\right)}{\left[216\left(s^{2}+3 s+2\right)\right]^{\frac{1}{q}}}\left(\frac{5}{72}\right)^{1-\frac{1}{q}}\left\{\left[\left(3^{-s} \times 2^{1-s}+3 s \times 2^{1-s}+3 \times 2^{-s}\right)\left|\psi^{\prime}\left(\lambda_{2}\right)\right|^{q}\right.\right. \\
& \left.+\left(5^{s+2} \times 3^{-s} \times 2^{1-s}-6 s \times 2^{-s}-21 \times 2^{-s}+6 s-24\right)\left|\psi^{\prime}\left(\lambda_{1}\right)\right|^{q}\right]^{\frac{1}{q}} \\
& +\left[\left(3^{-s} \times 2^{1-s}+3 s \times 2^{1-s}+3 \times 2^{-s}\right)\left|\psi^{\prime}\left(\lambda_{1}\right)\right|^{q}\right. \\
& \left.\left.+\left(5^{s+2} \times 3^{-s} \times 2^{1-s}-6 s \times 2^{-s}-21 \times 2^{-s}+6 s-24\right)\left|\psi^{\prime}\left(\lambda_{2}\right)\right|^{q}\right]^{\frac{1}{q}}\right\} .
\end{aligned}
$$

In [11], Sarikaya et al. gave the following results Simpson type inequalities for differentiable convex functions

Theorem 1.6. Let $\psi: \mathrm{I} \subset[0, \infty) \rightarrow \mathrm{R}$ be a differentiable mapping on $\mathrm{I}^{\circ}$ such that $\psi^{\prime} \in$ $\mathcal{L}\left[\lambda_{1}, \lambda_{2}\right]$, where $\lambda_{1}, \lambda_{2} \in \mathrm{I}^{\circ}$ with $\lambda_{1}<\lambda_{2}$. If $\left|\psi^{\prime}\right|$ is convex on $\left[\lambda_{1}, \lambda_{2}\right]$, then the following inequality holds:

$$
\begin{aligned}
& \left|\frac{1}{6}\left(\psi\left(\lambda_{1}\right)+4 \psi\left(\frac{\lambda_{1}+\lambda_{2}}{2}\right)+\psi\left(\lambda_{2}\right)\right)-\frac{1}{\lambda_{2}-\lambda_{1}} \int_{\lambda_{1}}^{\lambda_{2}} \psi(u) d u\right| \\
& \leqslant \frac{5\left(\lambda_{2}-\lambda_{1}\right)}{72}\left[\left|\psi^{\prime}\left(\lambda_{1}\right)\right|+\left|\psi^{\prime}\left(\lambda_{2}\right)\right|\right] .
\end{aligned}
$$

Theorem 1.7. Let $\psi: \mathrm{I} \subset[0, \infty) \rightarrow \mathrm{R}$ be a differentiable mapping on $\mathrm{I}^{\circ}$ such that $\psi^{\prime} \in$ $\mathcal{L}\left[\lambda_{1}, \lambda_{2}\right]$, where $\lambda_{1}, \lambda_{2} \in \mathrm{I}^{\circ}$ with $\lambda_{1}<\lambda_{2}$. If $\left|\psi^{\prime}\right|^{\mathrm{q}}$ is convex on $\left[\lambda_{1}, \lambda_{2}\right], \mathrm{q}>1$ with $\frac{1}{\mathrm{p}}+\frac{1}{\mathrm{q}}=1$, then the following inequality holds:

$$
\begin{aligned}
& \left|\frac{1}{6}\left(\psi\left(\lambda_{1}\right)+4 \psi\left(\frac{\lambda_{1}+\lambda_{2}}{2}\right)+\psi\left(\lambda_{2}\right)\right)-\frac{1}{\lambda_{2}-\lambda_{1}} \int_{\lambda_{1}}^{\lambda_{2}} \psi(u) d u\right| \\
\leqslant & \frac{\lambda_{2}-\lambda_{1}}{12}\left(\frac{1+2^{1+p}}{3(1+p)}\right)^{\frac{1}{p}}\left(\left(\frac{3\left|\psi^{\prime}\left(\lambda_{1}\right)\right|^{q}+\left|\psi^{\prime}\left(\lambda_{2}\right)\right|^{q}}{4}\right)^{\frac{1}{q}}+\left(\frac{\left|\psi^{\prime}\left(\lambda_{1}\right)\right|^{q}+3\left|\psi^{\prime}\left(\lambda_{2}\right)\right|^{q}}{4}\right)^{\frac{1}{q}}\right) .
\end{aligned}
$$

Theorem 1.8. Let $\psi: \mathrm{I} \subset[0, \infty) \rightarrow \mathrm{R}$ be a differentiable mapping on $\mathrm{I}^{\circ}$ such that $\psi^{\prime} \in$ $\mathcal{L}\left[\lambda_{1}, \lambda_{2}\right]$, where $\lambda_{1}, \lambda_{2} \in I^{\circ}$ with $\lambda_{1}<\lambda_{2}$. If $\left|\psi^{\prime}\right|^{q}$ is convex on $\left[\lambda_{1}, \lambda_{2}\right], q \geqslant 1$, then the following inequality holds:

$$
\begin{aligned}
& \left|\frac{1}{6}\left(\psi\left(\lambda_{1}\right)+4 \psi\left(\frac{\lambda_{1}+\lambda_{2}}{2}\right)+\psi\left(\lambda_{2}\right)\right)-\frac{1}{\lambda_{2}-\lambda_{1}} \int_{\lambda_{1}}^{\lambda_{2}} \psi(u) d u\right| \\
\leqslant & \frac{\lambda_{2}-\lambda_{1}}{72} 5^{1-\frac{1}{q}}\left(\left(\frac{61\left|\psi^{\prime}\left(\lambda_{1}\right)\right|^{\mathrm{q}}+29\left|\psi^{\prime}\left(\lambda_{2}\right)\right|^{\mathrm{q}}}{18}\right)^{\frac{1}{q}}+\left(\frac{29\left|\psi^{\prime}\left(\lambda_{1}\right)\right|^{\mathrm{q}}+61\left|\psi^{\prime}\left(\lambda_{2}\right)\right|^{\mathrm{q}}}{15}\right)^{\frac{1}{q}}\right) .
\end{aligned}
$$


In [12], Sarikaya et al. generalized the results given in [11] for differentiable s-convex functions.

Theorem 1.9. Let $\psi: \mathrm{I} \subset[0, \infty) \rightarrow \mathrm{R}$ be a differentiable mapping on $\mathrm{I}^{\circ}$ such that $\psi^{\prime} \in$ $\mathcal{L}\left[\lambda_{1}, \lambda_{2}\right]$, where $\lambda_{1}, \lambda_{2} \in I^{\circ}$ with $\lambda_{1}<\lambda_{2}$. If $\left|\psi^{\prime}\right|^{\mid}$is s-convex on $\left[\lambda_{1}, \lambda_{2}\right]$ for some fixed $s \in(0,1]$ and $\mathrm{q}>1$ with $\frac{1}{\mathrm{p}}+\frac{1}{\mathrm{q}}=1$, then the following inequality holds:

$$
\begin{aligned}
& \left|\frac{1}{6}\left(\psi\left(\lambda_{1}\right)+4 \psi\left(\frac{\lambda_{1}+\lambda_{2}}{2}\right)+\psi\left(\lambda_{2}\right)\right)-\frac{1}{\lambda_{2}-\lambda_{1}} \int_{\lambda_{1}}^{\lambda_{2}} \psi(u) d u\right| \\
& \leqslant \frac{\lambda_{2}-\lambda_{1}}{12}\left(\frac{1+2^{1+p}}{3(1+p)}\right)^{\frac{1}{p}} \\
& \quad \times\left(\left(\frac{\left|\psi^{\prime}\left(\lambda_{1}\right)\right|^{q}+\left|\psi^{\prime}\left(\frac{\lambda_{1}+\lambda_{2}}{2}\right)\right|^{q}}{s+1}\right)^{\frac{1}{q}}+\left(\frac{\left|\psi^{\prime}\left(\frac{\lambda_{1}+\lambda_{2}}{2}\right)\right|^{q}+\left|\psi^{\prime}\left(\lambda_{2}\right)\right|^{q}}{s+1}\right)^{\frac{1}{q}}\right) .
\end{aligned}
$$

Theorem 1.10. Let $\psi: \mathrm{I} \subset[0, \infty) \rightarrow \mathrm{R}$ be a differentiable mapping on $\mathrm{I}^{\circ}$ such that $\psi^{\prime} \in$ $\mathcal{L}\left[\lambda_{1}, \lambda_{2}\right]$, where $\lambda_{1}, \lambda_{2} \in \mathrm{I}^{\circ}$ with $\lambda_{1}<\lambda_{2}$. If $\left|\psi^{\prime}\right|^{9}$ is s-convex on $\left[\lambda_{1}, \lambda_{2}\right]$ for some fixed $s \in(0,1]$ and $\mathrm{q} \geqslant 1$, then the following inequality holds:

$$
\begin{aligned}
& \left|\frac{1}{6}\left(\psi\left(\lambda_{1}\right)+4 \psi\left(\frac{\lambda_{1}+\lambda_{2}}{2}\right)+\psi\left(\lambda_{2}\right)\right)-\frac{1}{\lambda_{2}-\lambda_{1}} \int_{\lambda_{1}}^{\lambda_{2}} \psi(u) d u\right| \\
\leqslant & \frac{\lambda_{2}-\lambda_{1}}{2}\left(\frac{5}{36}\right)^{1-\frac{1}{q}} \\
& \times\left(\left(\frac{(2 s+1) 3^{s+1}+2}{3 \times 6^{s+1}(s+1)(s+2)}\left|\psi^{\prime}\left(\lambda_{1}\right)\right|^{q}+\frac{2 \times 5^{s+2}+6^{s+1}(s-4)-3^{s+1}(2 s+7)}{3 \times 6^{s+1}(s+1)(s+2)}\left|\psi^{\prime}\left(\lambda_{2}\right)\right|^{q}\right)^{\frac{1}{q}}\right. \\
& \left.+\left(\frac{2 \times 5^{s+2}+6^{s+1}(s-4)-3^{s+1}(2 s+7)}{3 \times 6^{s+1}(s+1)(s+2)}\left|\psi^{\prime}\left(\lambda_{1}\right)\right|^{q}+\frac{(2 s+1) 3^{s+1}+2}{3 \times 6^{s+1}(s+1)(s+2)}\left|\psi^{\prime}\left(\lambda_{2}\right)\right|^{q}\right)^{\frac{1}{q}}\right) .
\end{aligned}
$$

In [13], Matloka gave the following weighted Simpson type inequalities.

Theorem 1.11. Let $\psi:\left[\lambda_{1}, \lambda_{2}\right] \rightarrow R$ be a differentiable mapping on $\left(\lambda_{1}, \lambda_{2}\right)$ such that $\psi^{\prime} \in$ $\mathcal{L}\left[\lambda_{1}, \lambda_{2}\right]$ with $\lambda_{1}<\lambda_{2}$ and $w:\left[\lambda_{1}, \lambda_{2}\right] \rightarrow \mathrm{R}$ be continuous and symmetric to $\frac{\lambda_{1}+\lambda_{2}}{2}$. If $\left|\psi^{\prime}\right|$ is s-convex on $\left[\lambda_{1}, \lambda_{2}\right]$ for some fixed $s \in(0,1]$, then the following inequality holds:

$$
\begin{aligned}
& \left|\frac{1}{6}\left(\psi\left(\lambda_{1}\right)+4 \psi\left(\frac{\lambda_{1}+\lambda_{2}}{2}\right)+\psi\left(\lambda_{2}\right)\right)\left(\int_{\lambda_{1}}^{\lambda_{2}} w(u) d u\right)-\int_{\lambda_{1}}^{\lambda_{2}} w(u) \psi(u) d u\right| \\
& \leqslant \frac{\left(\lambda_{2}-\lambda_{1}\right)^{2}}{1+s}\|w\|_{\left[\lambda_{1}, \lambda_{2}\right], \infty}\left(\left|\psi^{\prime}\left(\lambda_{1}\right)\right|+\left|\psi^{\prime}\left(\lambda_{2}\right)\right|\right) .
\end{aligned}
$$


Theorem 1.12. Let $\psi:\left[\lambda_{1}, \lambda_{2}\right] \rightarrow \mathrm{R}$ be a differentiable mapping on $\left(\lambda_{1}, \lambda_{2}\right)$ such that $\psi^{\prime} \in$ $\mathcal{L}\left[\lambda_{1}, \lambda_{2}\right]$ with $\lambda_{1}<\lambda_{2}$ and $w:\left[\lambda_{1}, \lambda_{2}\right] \rightarrow R$ be continuous and symmetric to $\frac{\lambda_{1}+\lambda_{2}}{2}$. If $\left|\psi^{\prime}\right|{ }^{\mathrm{q}}$ is s-convex on $\left[\lambda_{1}, \lambda_{2}\right]$ for some fixed $s \in(0,1]$ and $\mathrm{q}>1$ with $\frac{1}{\mathrm{p}}+\frac{1}{\mathrm{q}}=1$, then the following inequality holds:

$$
\begin{aligned}
& \left|\frac{1}{6}\left(\psi\left(\lambda_{1}\right)+4 \psi\left(\frac{\lambda_{1}+\lambda_{2}}{2}\right)+\psi\left(\lambda_{2}\right)\right)\left(\int_{\lambda_{1}}^{\lambda_{2}} w(u) d u\right)-\int_{\lambda_{1}}^{\lambda_{2}} w(u) \psi(u) d u\right| \\
& \leqslant \frac{\left(\lambda_{2}-\lambda_{1}\right)^{2}}{12}\|w\|_{\left[\lambda_{1}, \lambda_{2}\right], \infty}\left(\frac{1+2^{1+p}}{3(1+p)}\right)^{\frac{1}{p}}\left(\frac{1}{4}\right)^{\frac{1}{q}}\left(\frac{2}{s+1}\right)^{\frac{1}{q}} \\
& \quad \times\left\{\left(\left(\frac{1}{2}\right)^{s+1}\left|\psi^{\prime}\left(\lambda_{1}\right)\right|^{q}+\left[1-\left(\frac{1}{2}\right)^{s+1}\right]\left|\psi^{\prime}\left(\lambda_{2}\right)\right|^{q}\right)^{\frac{1}{q}}\right. \\
& \left.+\left(\left[1-\left(\frac{1}{2}\right)^{s+1}\right]\left|\psi^{\prime}\left(\lambda_{1}\right)\right|^{q}+\left(\frac{1}{2}\right)^{s+1}\left|\psi^{\prime}\left(\lambda_{2}\right)\right|^{q}\right)^{\frac{1}{q}}\right\} .
\end{aligned}
$$

In this paper, inspired by the above mentioned studies, we will establish a new identity and then applying it to derive new weighted Simpson type inequalities for sconvex functions in the second sense. In order to illustrate the efficient of our main results, some applications to special means and weighted Simpson quadrature formula will be obtain. At the end, a brief conclusion will be provided as well.

\section{Main results}

Lemma 2.1. Let $\psi: \mathrm{I} \subset \mathrm{R} \rightarrow \mathrm{R}$ be a differentiable function on $\mathrm{I}^{\circ}, \lambda_{1}, \lambda_{2} \in \mathrm{I}^{\circ}$ with $\lambda_{1}<\lambda_{2}$, and let $w:\left[\lambda_{1}, \lambda_{2}\right] \rightarrow R$ be symmetric with respect to $\frac{\lambda_{1}+\lambda_{2}}{2}$. If $\psi^{\prime}, w \in \mathcal{L}\left[\lambda_{1}, \lambda_{2}\right]$, then

$$
\begin{aligned}
& \frac{1}{6}\left(\psi\left(\lambda_{1}\right)+4 \psi\left(\frac{\lambda_{1}+\lambda_{2}}{2}\right)+\psi\left(\lambda_{2}\right)\right)\left(\int_{\lambda_{1}}^{\lambda_{2}} w(u) d u\right)-\int_{\lambda_{1}}^{\lambda_{2}} w(u) \psi(u) d u=\frac{3\left(\lambda_{2}-\lambda_{1}\right)^{2}}{4} \\
& \times\left[\int_{0}^{1} p_{1}(\chi) \psi^{\prime}\left(\chi \lambda_{1}+(1-\chi) \frac{\lambda_{1}+\lambda_{2}}{2}\right) d \chi+\int_{0}^{1} p_{2}(\chi) \psi^{\prime}\left(\chi \frac{\lambda_{1}+\lambda_{2}}{2}+(1-\chi) \lambda_{2}\right) d \chi\right]
\end{aligned}
$$

where

$$
p_{1}(\chi)=\frac{2}{9} \int_{0}^{1} w\left(\zeta \lambda_{1}+(1-\zeta) \frac{\lambda_{1}+\lambda_{2}}{2}\right) d \zeta-\frac{1}{3} \int_{0}^{\chi} w\left(\zeta \lambda_{1}+(1-\zeta) \frac{\lambda_{1}+\lambda_{2}}{2}\right) d \zeta
$$

and

$$
p_{2}(\chi)=\frac{1}{9} \int_{0}^{1} w\left(\zeta \frac{\lambda_{1}+\lambda_{2}}{2}+(1-\zeta) \lambda_{2}\right) d \zeta-\frac{1}{3} \int_{0}^{\chi} w\left(\zeta \frac{\lambda_{1}+\lambda_{2}}{2}+(1-\zeta) \lambda_{2}\right) d \zeta
$$


Proof. Integrating by parts and changing the variables, and using the symmetry of $w$, we obtain

$$
\begin{aligned}
& \int_{0}^{1} p_{1}(x) \psi^{\prime}\left(\chi \lambda_{1}+(1-\chi) \frac{\lambda_{1}+\lambda_{2}}{2}\right) d x \\
& =\int_{0}^{1}\left(\frac{2}{9} \int_{0}^{1} w\left(\zeta \lambda_{1}+(1-\zeta) \frac{\lambda_{1}+\lambda_{2}}{2}\right) d \zeta-\frac{1}{3} \int_{0}^{\chi} w\left(\zeta \lambda_{1}+(1-\zeta) \frac{\lambda_{1}+\lambda_{2}}{2}\right) d \zeta\right) \\
& \times \psi^{\prime}\left(\chi \lambda_{1}+(1-\chi) \frac{\lambda_{1}+\lambda_{2}}{2}\right) d \chi \\
& =\frac{2}{\lambda_{2}-\lambda_{1}}\left(\frac{2}{9} \int_{0}^{1} w\left(\zeta \lambda_{1}+(1-\zeta) \frac{\lambda_{1}+\lambda_{2}}{2}\right) d \zeta-\frac{1}{3} \int_{0}^{\chi} w\left(\zeta \lambda_{1}+(1-\zeta) \frac{\lambda_{1}+\lambda_{2}}{2}\right) d \zeta\right) \\
& \times\left.\psi\left(\chi \lambda_{1}+(1-\chi) \frac{\lambda_{1}+\lambda_{2}}{2}\right)\right|_{\chi=0} ^{\chi=1} \\
& -\frac{2}{3\left(\lambda_{2}-\lambda_{1}\right)} \int_{0}^{1} w\left(\chi \lambda_{1}+(1-\chi) \frac{\lambda_{1}+\lambda_{2}}{2}\right) \psi\left(\chi \lambda_{1}+(1-\chi) \frac{\lambda_{1}+\lambda_{2}}{2}\right) d \chi \\
& =\frac{2}{9\left(\lambda_{2}-\lambda_{1}\right)}\left(\int_{0}^{1} w\left(\zeta \lambda_{1}+(1-\zeta) \frac{\lambda_{1}+\lambda_{2}}{2}\right) d \zeta\right) \psi\left(\lambda_{1}\right) \\
& +\frac{4}{9\left(\lambda_{2}-\lambda_{1}\right)}\left(\int_{0}^{1} w\left(\zeta \lambda_{1}+(1-\zeta) \frac{\lambda_{1}+\lambda_{2}}{2}\right) d \zeta\right) \psi\left(\frac{\lambda_{1}+\lambda_{2}}{2}\right) \\
& -\frac{2}{3\left(\lambda_{2}-\lambda_{1}\right)} \int_{0}^{1} w\left(\chi \lambda_{1}+(1-\chi) \frac{\lambda_{1}+\lambda_{2}}{2}\right) \psi\left(\chi \lambda_{1}+(1-\chi) \frac{\lambda_{1}+\lambda_{2}}{2}\right) d \chi \\
& =\frac{4}{9\left(\lambda_{2}-\lambda_{1}\right)^{2}}\left(\int_{\lambda_{1}}^{\frac{\lambda_{1}+\lambda_{2}}{2}} w(u) d u\right) \psi\left(\lambda_{1}\right)+\frac{8}{9\left(\lambda_{2}-\lambda_{1}\right)^{2}}\left(\int_{\lambda_{1}}^{\frac{\lambda_{1}+\lambda_{2}}{2}} w(u) d u\right) \psi\left(\frac{\lambda_{1}+\lambda_{2}}{2}\right) \\
& -\frac{4}{3\left(\lambda_{2}-\lambda_{1}\right)^{2}} \int_{\lambda_{1}}^{\frac{\lambda_{1}+\lambda_{2}}{2}} w(u) \psi(u) d u
\end{aligned}
$$




$$
\begin{aligned}
= & \frac{2}{9\left(\lambda_{2}-\lambda_{1}\right)^{2}}\left(\int_{\lambda_{1}}^{\lambda_{2}} w(u) d u\right) \psi\left(\lambda_{1}\right)+\frac{4}{9\left(\lambda_{2}-\lambda_{1}\right)^{2}}\left(\int_{\lambda_{1}}^{\lambda_{2}} w(u) d u\right) \psi\left(\frac{\lambda_{1}+\lambda_{2}}{2}\right) \\
& -\frac{4}{3\left(\lambda_{2}-\lambda_{1}\right)^{2}} \int_{\lambda_{1}}^{\frac{\lambda_{1}+\lambda_{2}}{2}} w(u) \psi(u) d u .
\end{aligned}
$$

Similarly, we have

$$
\begin{aligned}
& \int_{0}^{1} p_{2}(\chi) \psi^{\prime}\left(\chi \lambda_{1}+(1-\chi) x\right) d \chi \\
& =\int_{0}^{1}\left(\frac{1}{9} \int_{0}^{1} w\left(\zeta \frac{\lambda_{1}+\lambda_{2}}{2}+(1-\zeta) \lambda_{2}\right) d \zeta-\frac{1}{3} \int_{0}^{\chi} w\left(\zeta \frac{\lambda_{1}+\lambda_{2}}{2}+(1-\zeta) \lambda_{2}\right) d \zeta\right) \\
& \times \psi^{\prime}\left(\chi \frac{\lambda_{1}+\lambda_{2}}{2}+(1-\chi) \lambda_{2}\right) d \chi \\
& =-\frac{2}{\lambda_{2}-\lambda_{1}}\left(\frac{1}{9} \int_{0}^{1} w\left(\zeta \frac{\lambda_{1}+\lambda_{2}}{2}+(1-\zeta) \lambda_{2}\right) d \zeta-\frac{1}{3} \int_{0}^{x} w\left(\zeta \frac{\lambda_{1}+\lambda_{2}}{2}+(1-\zeta) \lambda_{2}\right) d \zeta\right) \\
& \times\left.\psi\left(\chi \frac{\lambda_{1}+\lambda_{2}}{2}+(1-\chi) \lambda_{2}\right)\right|_{\chi=0} ^{\chi=1} \\
& -\frac{2}{3\left(\lambda_{2}-\lambda_{1}\right)} \int_{0}^{1} w\left(\chi \frac{\lambda_{1}+\lambda_{2}}{2}+(1-\chi) \lambda_{2}\right) \psi\left(\chi \frac{\lambda_{1}+\lambda_{2}}{2}+(1-\chi) \lambda_{2}\right) d \chi \\
& =\frac{4}{9\left(\lambda_{2}-\lambda_{1}\right)}\left(\int_{0}^{1} w\left(\zeta \frac{\lambda_{1}+\lambda_{2}}{2}+(1-\zeta) \lambda_{2}\right) d \zeta\right) \psi\left(\frac{\lambda_{1}+\lambda_{2}}{2}\right) \\
& +\frac{2}{9\left(\lambda_{2}-\lambda_{1}\right)}\left(\int_{0}^{1} w\left(\zeta \frac{\lambda_{1}+\lambda_{2}}{2}+(1-\zeta) \lambda_{2}\right) d \zeta\right) \psi\left(\lambda_{2}\right) \\
& -\frac{2}{3\left(\lambda_{2}-\lambda_{1}\right)} \int_{0}^{1} w\left(\chi \frac{\lambda_{1}+\lambda_{2}}{2}+(1-\chi) \lambda_{2}\right) \psi\left(\chi \frac{\lambda_{1}+\lambda_{2}}{2}+(1-\chi) \lambda_{2}\right) d \chi \\
& =\frac{8}{9\left(\lambda_{2}-\lambda_{1}\right)^{2}}\left(\int_{\frac{\lambda_{1}+\lambda_{2}}{2}}^{\lambda_{2}} w(u) d u\right) \psi\left(\frac{\lambda_{1}+\lambda_{2}}{2}\right)+\frac{4}{9\left(\lambda_{2}-\lambda_{1}\right)^{2}}\left(\int_{\frac{\lambda_{1}+\lambda_{2}}{2}}^{\lambda_{2}} w(u) d u\right) \psi\left(\lambda_{2}\right)
\end{aligned}
$$




$$
\begin{aligned}
& -\frac{4}{3\left(\lambda_{2}-\lambda_{1}\right)^{2}} \int_{\frac{\lambda_{1}+\lambda_{2}}{2}}^{\lambda_{2}} w(u) \psi(u) d u \\
= & \frac{4}{9\left(\lambda_{2}-\lambda_{1}\right)^{2}}\left(\int_{\lambda_{1}}^{\lambda_{2}} w(u) d u\right) \psi\left(\frac{\lambda_{1}+\lambda_{2}}{2}\right)+\frac{2}{9\left(\lambda_{2}-\lambda_{1}\right)^{2}}\left(\int_{\lambda_{1}}^{\lambda_{2}} w(u) d u\right) \psi\left(\lambda_{2}\right) \\
& -\frac{4}{3\left(\lambda_{2}-\lambda_{1}\right)^{2}} \int_{\frac{\lambda_{1}+\lambda_{2}}{2}}^{\lambda_{2}} w(u) \psi(u) d u .
\end{aligned}
$$

Summing (2.3) and (2.4), and then multiplying the resulting equality by factor $\frac{3\left(\lambda_{2}-\lambda_{1}\right)^{2}}{4}$, we get the desired result.

Theorem 2.2. Let $\psi:\left[\lambda_{1}, \lambda_{2}\right] \rightarrow \Re$ be a differentiable function on $\left(\lambda_{1}, \lambda_{2}\right)$ such that $\psi^{\prime} \in$ $\mathcal{L}\left[\lambda_{1}, \lambda_{2}\right]$ with $0 \leqslant \lambda_{1}<\lambda_{2}$, and let $w:\left[\lambda_{1}, \lambda_{2}\right] \rightarrow R$ be continuous and symmetric function with respect to $\frac{\lambda_{1}+\lambda_{2}}{2}$. If $\left|\psi^{\prime}\right|$ is s-convex in the second sense for some fixed $s \in(0,1]$, then

$$
\begin{aligned}
& \left|\frac{1}{6}\left(\psi\left(\lambda_{1}\right)+4 \psi\left(\frac{\lambda_{1}+\lambda_{2}}{2}\right)+\psi\left(\lambda_{2}\right)\right)\left(\int_{\lambda_{1}}^{\lambda_{2}} w(u) d u\right)-\int_{\lambda_{1}}^{\lambda_{2}} w(u) \psi(u) d u\right| \\
& \leqslant \\
& \leqslant \frac{3\left(\lambda_{2}-\lambda_{1}\right)^{2}}{4(1+s)(2+s)}\|w\|_{\left[\lambda_{1}, \lambda_{2}\right], \infty}\left(\left(\frac{s-1}{9}+\left(\frac{2}{3}\right)^{3+s}\right)\left|\psi^{\prime}\left(\lambda_{1}\right)\right|\right. \\
& \left.\quad+2\left(\frac{1+2 s}{9}+2\left(\frac{1}{3}\right)^{3+s}\right)\left|\psi^{\prime}\left(\frac{\lambda_{1}+\lambda_{2}}{2}\right)\right|+\left(\frac{s-1}{9}+\left(\frac{2}{3}\right)^{3+s}\right)\left|\psi^{\prime}\left(\lambda_{2}\right)\right|\right) .
\end{aligned}
$$

Proof. From Lemma 2.1, and properties of modulus, we have

$$
\begin{aligned}
& \left|\frac{1}{6}\left(\psi\left(\lambda_{1}\right)+4 \psi\left(\frac{\lambda_{1}+\lambda_{2}}{2}\right)+\psi\left(\lambda_{2}\right)\right)\left(\int_{\lambda_{1}}^{\lambda_{2}} w(u) d u\right)-\int_{\lambda_{1}}^{\lambda_{2}} w(u) \psi(u) d u\right| \\
& \leqslant \\
& \leqslant \frac{3\left(\lambda_{2}-\lambda_{1}\right)^{2}}{4} \int_{0}^{1}\left|p_{1}(\chi)\right|\left|\psi^{\prime}\left(\chi \lambda_{1}+(1-\chi) \frac{\lambda_{1}+\lambda_{2}}{2}\right)\right| d \chi \\
& \quad+\frac{3\left(\lambda_{2}-\lambda_{1}\right)^{2}}{4} \int_{0}^{1}\left|p_{2}(\chi)\right|\left|\psi^{\prime}\left(\chi \frac{\lambda_{1}+\lambda_{2}}{2}+(1-\chi) \lambda_{2}\right)\right| d x .
\end{aligned}
$$

Since $\left|\psi^{\prime}\right|$ is s-convex, we get

$$
\begin{aligned}
& \left|\psi^{\prime}\left(\chi \lambda_{1}+(1-\chi) \frac{\lambda_{1}+\lambda_{2}}{2}\right)\right| \leqslant \chi^{s}\left|\psi^{\prime}\left(\lambda_{1}\right)\right|+(1-\chi)^{s}\left|\psi^{\prime}\left(\frac{\lambda_{1}+\lambda_{2}}{2}\right)\right|, \\
& \left|\psi^{\prime}\left(\chi \frac{\lambda_{1}+\lambda_{2}}{2}+(1-\chi) \lambda_{2}\right)\right| \leqslant \chi^{s}\left|\psi^{\prime}\left(\frac{\lambda_{1}+\lambda_{2}}{2}\right)\right|+(1-\chi)^{s}\left|\psi^{\prime}\left(\lambda_{2}\right)\right| .
\end{aligned}
$$


Using (2.1), (2.2), (2.6) and (2.7) in (2.5), we obtain

$$
\begin{aligned}
& \left|\frac{1}{6}\left(\psi\left(\lambda_{1}\right)+4 \psi\left(\frac{\lambda_{1}+\lambda_{2}}{2}\right)+\psi\left(\lambda_{2}\right)\right)\left(\int_{\lambda_{1}}^{\lambda_{2}} w(u) d u\right)-\int_{\lambda_{1}}^{\lambda_{2}} w(u) \psi(u) d u\right| \\
& \leqslant \frac{3\left(\lambda_{2}-\lambda_{1}\right)^{2}}{4} \int_{0}^{1}\left|\frac{2}{9} \int_{0}^{1} w\left(\zeta \lambda_{1}+(1-\zeta) \frac{\lambda_{1}+\lambda_{2}}{2}\right) \mathrm{d} \zeta-\frac{1}{3} \int_{0}^{\chi} w\left(\zeta \lambda_{1}+(1-\zeta) \frac{\lambda_{1}+\lambda_{2}}{2}\right) \mathrm{d} \zeta\right| \\
& \times\left(\chi^{s}\left|\psi^{\prime}\left(\lambda_{1}\right)\right|+(1-\chi)^{s}\left|\psi^{\prime}\left(\frac{\lambda_{1}+\lambda_{2}}{2}\right)\right|\right) d \chi \\
& +\frac{3\left(\lambda_{2}-\lambda_{1}\right)^{2}}{4} \int_{0}^{1}\left|\frac{1}{9} \int_{0}^{1} w\left(\zeta \frac{\lambda_{1}+\lambda_{2}}{2}+(1-\zeta) \lambda_{2}\right) d \zeta-\frac{1}{3} \int_{0}^{x} w\left(\zeta \frac{\lambda_{1}+\lambda_{2}}{2}+(1-\zeta) \lambda_{2}\right) d \zeta\right| \\
& \times\left(\chi^{s}\left|\psi^{\prime}\left(\frac{\lambda_{1}+\lambda_{2}}{2}\right)\right|+(1-\chi)^{s}\left|\psi^{\prime}\left(\lambda_{2}\right)\right|\right) d x \\
& \leqslant \frac{3\left(\lambda_{2}-\lambda_{1}\right)^{2}}{4}\|w\|_{\left[\lambda_{1}, \lambda_{2}\right], \infty} \int_{0}^{1}\left|\frac{2}{9} \int_{0}^{1} \mathrm{~d} \zeta-\frac{1}{3} \int_{0}^{x} \mathrm{~d} \zeta\right|\left(\chi^{\mathrm{s}}\left|\psi^{\prime}\left(\lambda_{1}\right)\right|+(1-\chi)^{\mathrm{s}}\left|\psi^{\prime}\left(\frac{\lambda_{1}+\lambda_{2}}{2}\right)\right|\right) \mathrm{d} \chi \\
& +\frac{3\left(\lambda_{2}-\lambda_{1}\right)^{2}}{4}\|w\|_{\left[\lambda_{1}, \lambda_{2}\right], \infty} \int_{0}^{1}\left|\frac{1}{9} \int_{0}^{1} d \zeta-\frac{1}{3} \int_{0}^{\chi} d \zeta\right|\left(\chi^{s}\left|\psi^{\prime}\left(\frac{\lambda_{1}+\lambda_{2}}{2}\right)\right|+(1-\chi)^{s}\left|\psi^{\prime}\left(\lambda_{2}\right)\right|\right) d \chi \\
& =\frac{3\left(\lambda_{2}-\lambda_{1}\right)^{2}}{4}\|w\|_{\left[\lambda_{1}, \lambda_{2}\right], \infty} \int_{0}^{1}\left|\frac{2}{9}-\frac{1}{3} \chi\right|\left(\chi^{s}\left|\psi^{\prime}\left(\lambda_{1}\right)\right|+(1-\chi)^{s}\left|\psi^{\prime}\left(\frac{\lambda_{1}+\lambda_{2}}{2}\right)\right|\right) d \chi \\
& +\frac{3\left(\lambda_{2}-\lambda_{1}\right)^{2}}{4}\|w\|_{\left[\lambda_{1}, \lambda_{2}\right], \infty} \int_{0}^{1}\left|\frac{1}{9}-\frac{1}{3} \chi\right|\left(\chi^{s}\left|\psi^{\prime}\left(\frac{\lambda_{1}+\lambda_{2}}{2}\right)\right|+(1-\chi)^{s}\left|\psi^{\prime}\left(\lambda_{2}\right)\right|\right) d \chi \\
& =\frac{3\left(\lambda_{2}-\lambda_{1}\right)^{2}}{4}\|w\|_{\left[\lambda_{1}, \lambda_{2}\right], \infty}\left(\left|\psi^{\prime}\left(\lambda_{1}\right)\right| \int_{0}^{1}\left|\frac{2}{9}-\frac{1}{3} \chi\right| \chi^{s} \mathrm{~d} \chi+\left|\psi^{\prime}\left(\frac{\lambda_{1}+\lambda_{2}}{2}\right)\right| \int_{0}^{1}\left|\frac{2}{9}-\frac{1}{3} \chi\right|(1-\chi)^{s} \mathrm{~d} \chi\right. \\
& \left.+\left|\psi^{\prime}\left(\frac{\lambda_{1}+\lambda_{2}}{2}\right)\right| \int_{0}^{1}\left|\frac{1}{9}-\frac{1}{3} \chi\right| \chi^{s} \mathrm{~d} \chi+\left|\psi^{\prime}\left(\lambda_{2}\right)\right| \int_{0}^{1}\left|\frac{1}{9}-\frac{1}{3} \chi\right|(1-\chi)^{s} \mathrm{~d} \chi\right) \\
& =\frac{3\left(\lambda_{2}-\lambda_{1}\right)^{2}}{4(1+s)(2+s)}\|w\|_{\left[\lambda_{1}, \lambda_{2}\right], \infty}\left(\left(\frac{s-1}{9}+\left(\frac{2}{3}\right)^{3+s}\right)\left|\psi^{\prime}\left(\lambda_{1}\right)\right|\right. \\
& \left.+2\left(\frac{1+2 s}{9}+2\left(\frac{1}{3}\right)^{3+s}\right)\left|\psi^{\prime}\left(\frac{\lambda_{1}+\lambda_{2}}{2}\right)\right|+\left(\frac{s-1}{9}+\left(\frac{2}{3}\right)^{3+s}\right)\left|\psi^{\prime}\left(\lambda_{2}\right)\right|\right),
\end{aligned}
$$


where we have used the fact that

$$
\begin{aligned}
\int_{0}^{1}\left|\frac{2}{9}-\frac{1}{3} \chi\right| \chi^{s} \mathrm{~d} \chi & =\int_{0}^{1}\left|\frac{1}{9}-\frac{1}{3} \chi\right|(1-\chi)^{s} \mathrm{~d} \chi \\
& =\frac{1}{(1+s)(2+s)}\left(\frac{s-1}{9}+\left(\frac{2}{3}\right)^{3+s}\right)
\end{aligned}
$$

and

$$
\begin{aligned}
\int_{0}^{1}\left|\frac{1}{9}-\frac{1}{3} \chi\right| \chi^{s} \mathrm{~d} x & =\int_{0}^{1}\left|\frac{2}{9}-\frac{1}{3} \chi\right|(1-x)^{s} \mathrm{~d} \chi \\
& =\frac{1}{(1+s)(2+s)}\left(\frac{1+2 s}{9}+2\left(\frac{1}{3}\right)^{3+s}\right) .
\end{aligned}
$$

The proof is completed.

Corollary 2.3. In Theorem 2.2 , if we take $w(u)=\frac{1}{\lambda_{2}-\lambda_{1}}$, we have

$$
\begin{aligned}
& \left|\frac{1}{6}\left(\psi\left(\lambda_{1}\right)+4 \psi\left(\frac{\lambda_{1}+\lambda_{2}}{2}\right)+\psi\left(\lambda_{2}\right)\right)-\frac{1}{\lambda_{2}-\lambda_{1}} \int_{\lambda_{1}}^{\lambda_{2}} \psi(u) d u\right| \\
& \leqslant \frac{3\left(\lambda_{2}-\lambda_{1}\right)}{4(1+s)(2+s)}\left(\left(\frac{s-1}{9}+\left(\frac{2}{3}\right)^{3+s}\right)\left|\psi^{\prime}\left(\lambda_{1}\right)\right|\right. \\
& \left.\quad+2\left(\frac{1+2 s}{9}+2\left(\frac{1}{3}\right)^{3+s}\right)\left|\psi^{\prime}\left(\frac{\lambda_{1}+\lambda_{2}}{2}\right)\right|+\left(\frac{s-1}{9}+\left(\frac{2}{3}\right)^{3+s}\right)\left|\psi^{\prime}\left(\lambda_{2}\right)\right|\right) .
\end{aligned}
$$

Corollary 2.4. In Theorem 2.2, if we take $s=1$, we get

$$
\begin{aligned}
& \left|\frac{1}{6}\left(\psi\left(\lambda_{1}\right)+4 \psi\left(\frac{\lambda_{1}+\lambda_{2}}{2}\right)+\psi\left(\lambda_{2}\right)\right)\left(\int_{\lambda_{1}}^{\lambda_{2}} w(u) d u\right)-\int_{\lambda_{1}}^{\lambda_{2}} w(u) \psi(u) d u\right| \\
& \leqslant \frac{\left(\lambda_{2}-\lambda_{1}\right)^{2}}{324}\|w\|_{\left[\lambda_{1}, \lambda_{2}\right], \infty}\left(8\left|\psi^{\prime}\left(\lambda_{1}\right)\right|+29\left|\psi^{\prime}\left(\frac{\lambda_{1}+\lambda_{2}}{2}\right)\right|+8\left|\psi^{\prime}\left(\lambda_{2}\right)\right|\right) .
\end{aligned}
$$

Moreover, if we choose $w(u)=\frac{1}{\lambda_{2}-\lambda_{1}}$, we obtain

$$
\begin{aligned}
& \left|\frac{1}{6}\left(\psi\left(\lambda_{1}\right)+4 \psi\left(\frac{\lambda_{1}+\lambda_{2}}{2}\right)+\psi\left(\lambda_{2}\right)\right)-\frac{1}{\lambda_{2}-\lambda_{1}} \int_{\lambda_{1}}^{\lambda_{2}} \psi(u) d u\right| \\
& \leqslant \frac{\lambda_{2}-\lambda_{1}}{324}\left(8\left|\psi^{\prime}\left(\lambda_{1}\right)\right|+29\left|\psi^{\prime}\left(\frac{\lambda_{1}+\lambda_{2}}{2}\right)\right|+8\left|\psi^{\prime}\left(\lambda_{2}\right)\right|\right) .
\end{aligned}
$$


Corollary 2.5. In Corollary 2.4, using the convexity of $\left|\psi^{\prime}\right|$, we have

$$
\begin{aligned}
& \left|\frac{1}{6}\left(\psi\left(\lambda_{1}\right)+4 \psi\left(\frac{\lambda_{1}+\lambda_{2}}{2}\right)+\psi\left(\lambda_{2}\right)\right)\left(\int_{\lambda_{1}}^{\lambda_{2}} w(u) d u\right)-\int_{\lambda_{1}}^{\lambda_{2}} w(u) \psi(u) d u\right| \\
& \leqslant \frac{5\left(\lambda_{2}-\lambda_{1}\right)^{2}}{72}\|w\|_{\left[\lambda_{1}, \lambda_{2}\right], \infty}\left(\left|\psi^{\prime}\left(\lambda_{1}\right)\right|+\left|\psi^{\prime}\left(\lambda_{2}\right)\right|\right) .
\end{aligned}
$$

Moreover, if we choose $w(u)=\frac{1}{\lambda_{2}-\lambda_{1}}$, we obtain Theorem 1.6 from [11], also Corollary 1 from [10].

Theorem 2.6. Let $\psi:\left[\lambda_{1}, \lambda_{2}\right] \rightarrow \mathrm{R}$ be a differentiable function on $\left(\lambda_{1}, \lambda_{2}\right)$ such that $\psi^{\prime} \in$ $\mathcal{L}\left[\lambda_{1}, \lambda_{2}\right]$ with $0 \leqslant \lambda_{1}<\lambda_{2}$, and let $w:\left[\lambda_{1}, \lambda_{2}\right] \rightarrow R$ be continuous and symmetric function with respect to $\frac{\lambda_{1}+\lambda_{2}}{2}$. If $\left|\psi^{\prime}\right|^{q}$ is s-convex in the second sense for some fixed $s \in(0,1]$, where $\mathrm{q}>1$ with $\frac{1}{\mathrm{p}}+\frac{1}{\mathrm{q}}=1$, then

$$
\begin{aligned}
& \left|\frac{1}{6}\left(\psi\left(\lambda_{1}\right)+4 \psi\left(\frac{\lambda_{1}+\lambda_{2}}{2}\right)+\psi\left(\lambda_{2}\right)\right)\left(\int_{\lambda_{1}}^{\lambda_{2}} w(u) d u\right)-\int_{\lambda_{1}}^{\lambda_{2}} w(u) \psi(u) d u\right| \\
\leqslant & \frac{\left(\lambda_{2}-\lambda_{1}\right)^{2}}{12}\|w\|_{\left[\lambda_{1}, \lambda_{2}\right], \infty}\left(\frac{1+2^{1+p}}{3(1+p)}\right)^{\frac{1}{p}}\left(\frac{1}{1+s}\right)^{\frac{1}{q}} \\
& \times\left(\left(\left|\psi^{\prime}\left(\lambda_{1}\right)\right|^{\mathrm{q}}+\left|\psi^{\prime}\left(\frac{\lambda_{1}+\lambda_{2}}{2}\right)\right|^{q}\right)^{\frac{1}{q}}+\left(\left|\psi^{\prime}\left(\frac{\lambda_{1}+\lambda_{2}}{2}\right)\right|^{q}+\left|\psi^{\prime}\left(\lambda_{2}\right)\right|^{q}\right)^{\frac{1}{q}}\right) .
\end{aligned}
$$

Proof. From Lemma 2.1, properties of modulus, Hölder inequality, and s-convexity of $\left|\psi^{\prime}\right|^{\mathrm{q}}$, we have

$$
\begin{aligned}
& \left|\frac{1}{6}\left(\psi\left(\lambda_{1}\right)+4 \psi\left(\frac{\lambda_{1}+\lambda_{2}}{2}\right)+\psi\left(\lambda_{2}\right)\right)\left(\int_{\lambda_{1}}^{\lambda_{2}} w(u) d u\right)-\int_{\lambda_{1}}^{\lambda_{2}} w(u) \psi(u) d u\right| \\
& \leqslant \frac{3\left(\lambda_{2}-\lambda_{1}\right)^{2}}{4}\left(\int_{0}^{1}\left|p_{1}(x)\right|^{p} d x\right)^{\frac{1}{p}}\left(\int_{0}^{1}\left|\psi^{\prime}\left(x \lambda_{1}+(1-\chi) \frac{\lambda_{1}+\lambda_{2}}{2}\right)\right|^{q} d x\right)^{\frac{1}{q}} \\
& +\frac{3\left(\lambda_{2}-\lambda_{1}\right)^{2}}{4}\left(\int_{0}^{1}\left|p_{2}(\chi)\right|^{p} d \chi\right)^{\frac{1}{p}}\left(\int_{0}^{1}\left|\psi^{\prime}\left(\chi \frac{\lambda_{1}+\lambda_{2}}{2}+(1-\chi) \lambda_{2}\right)\right|^{q} d \chi\right)^{\frac{1}{q}} \\
& \leqslant \frac{3\left(\lambda_{2}-\lambda_{1}\right)^{2}}{4}\left(\int_{0}^{1}\left|\frac{2}{9} \int_{0}^{1} w\left(\zeta \lambda_{1}+(1-\zeta) \frac{\lambda_{1}+\lambda_{2}}{2}\right) d \zeta-\frac{1}{3} \int_{0}^{x} w\left(\zeta \lambda_{1}+(1-\zeta) \frac{\lambda_{1}+\lambda_{2}}{2}\right) d \zeta\right|^{p} d \chi\right)^{\frac{1}{p}}
\end{aligned}
$$




$$
\begin{aligned}
& \times\left(\int_{0}^{1}\left(\chi^{\mathrm{s}}\left|\psi^{\prime}\left(\lambda_{1}\right)\right|^{\mathrm{q}}+(1-\chi)^{\mathrm{s}}\left|\psi^{\prime}\left(\frac{\lambda_{1}+\lambda_{2}}{2}\right)\right|^{\mathrm{q}}\right) \mathrm{d} \chi\right)^{\frac{1}{q}} \\
& +\frac{3\left(\lambda_{2}-\lambda_{1}\right)^{2}}{4}\left(\int_{0}^{1}\left|\frac{1}{9} \int_{0}^{1} w\left(\zeta \frac{\lambda_{1}+\lambda_{2}}{2}+(1-\zeta) \lambda_{2}\right) d \zeta-\frac{1}{3} \int_{0}^{x} w\left(\zeta \frac{\lambda_{1}+\lambda_{2}}{2}+(1-\zeta) \lambda_{2}\right) d \zeta\right|^{p} d x\right)^{\frac{1}{p}} \\
& \times\left(\int_{0}^{1}\left(\chi^{s}\left|\psi^{\prime}\left(\frac{\lambda_{1}+\lambda_{2}}{2}\right)\right|^{q}+(1-\chi)^{s}\left|\psi^{\prime}\left(\lambda_{2}\right)\right|^{q}\right) d x\right)^{\frac{1}{q}} \\
& \leqslant \frac{3\left(\lambda_{2}-\lambda_{1}\right)^{2}}{4(1+s)^{\frac{1}{q}}}\|w\|_{\left[\lambda_{1}, \lambda_{2}\right], \infty}\left(\int_{0}^{1}\left|\frac{2}{9}-\frac{1}{3} \chi\right|^{p} d \chi\right)^{\frac{1}{p}}\left(\left|\psi^{\prime}\left(\lambda_{1}\right)\right|^{q}+\left|\psi^{\prime}\left(\frac{\lambda_{1}+\lambda_{2}}{2}\right)\right|^{q}\right)^{\frac{1}{q}} \\
& +\frac{3\left(\lambda_{2}-\lambda_{1}\right)^{2}}{4(1+s)^{\frac{1}{q}}}\|w\|_{\left[\lambda_{1}, \lambda_{2}\right], \infty}\left(\int_{0}^{1}\left|\frac{1}{9}-\frac{1}{3} \chi\right|^{p} \mathrm{~d} \chi\right)^{\frac{1}{p}}\left(\left|\psi^{\prime}\left(\frac{\lambda_{1}+\lambda_{2}}{2}\right)\right|^{q}+\left|\psi^{\prime}\left(\lambda_{2}\right)\right|^{q}\right)^{\frac{1}{q}} \\
& =\frac{\left(\lambda_{2}-\lambda_{1}\right)^{2}}{12}\|w\|_{\left[\lambda_{1}, \lambda_{2}\right], \infty}\left(\frac{1+2^{1+p}}{3(1+p)}\right)^{\frac{1}{p}}\left(\frac{1}{1+s}\right)^{\frac{1}{q}} \\
& \times\left(\left(\left|\psi^{\prime}\left(\lambda_{1}\right)\right|^{q}+\left|\psi^{\prime}\left(\frac{\lambda_{1}+\lambda_{2}}{2}\right)\right|^{q}\right)^{\frac{1}{q}}+\left(\left|\psi^{\prime}\left(\frac{\lambda_{1}+\lambda_{2}}{2}\right)\right|^{q}+\left|\psi^{\prime}\left(\lambda_{2}\right)\right|^{q}\right)^{\frac{1}{q}}\right) \text {. }
\end{aligned}
$$

The proof is completed.

Remark 2.7. In Theorem 2.6, if we take $w(u)=\frac{1}{\lambda_{2}-\lambda_{1}}$, we obtain Theorem 1.9 from [12].

Corollary 2.8. In Theorem 2.6 , if we take $\mathrm{s}=1$, we have

$$
\begin{aligned}
& \left|\frac{1}{6}\left(\psi\left(\lambda_{1}\right)+4 \psi\left(\frac{\lambda_{1}+\lambda_{2}}{2}\right)+\psi\left(\lambda_{2}\right)\right)\left(\int_{\lambda_{1}}^{\lambda_{2}} w(u) d u\right)-\int_{\lambda_{1}}^{\lambda_{2}} w(u) \psi(u) d u\right| \\
\leqslant & \frac{\left(\lambda_{2}-\lambda_{1}\right)^{2}}{12}\|w\|_{\left[\lambda_{1}, \lambda_{2}\right], \infty}\left(\frac{1+2^{1+p}}{3(1+p)}\right)^{\frac{1}{p}}\left(\frac{1}{2}\right)^{\frac{1}{q}} \\
& \left(\left(\left|\psi^{\prime}\left(\lambda_{1}\right)\right|^{q}+\left|\psi^{\prime}\left(\frac{\lambda_{1}+\lambda_{2}}{2}\right)\right|^{q}\right)^{\frac{1}{q}}+\left(\left|\psi^{\prime}\left(\frac{\lambda_{1}+\lambda_{2}}{2}\right)\right|^{q}+\left|\psi^{\prime}\left(\lambda_{2}\right)\right|^{q}\right)^{\frac{1}{q}}\right) .
\end{aligned}
$$

Moreover, if we choose $w(u)=\frac{1}{\lambda_{2}-\lambda_{1}}$, we get Corollary 3 from [12]. 
Corollary 2.9. In Corollary 2.8, using the convexity of $\left|\psi^{\prime}\right|^{\mathrm{q}}$, we obtain

$$
\begin{aligned}
& \left|\frac{1}{6}\left(\psi\left(\lambda_{1}\right)+4 \psi\left(\frac{\lambda_{1}+\lambda_{2}}{2}\right)+\psi\left(\lambda_{2}\right)\right)\left(\int_{\lambda_{1}}^{\lambda_{2}} w(u) d u\right)-\int_{\lambda_{1}}^{\lambda_{2}} w(u) \psi(u) d u\right| \\
\leqslant & \frac{\lambda_{2}-\lambda_{1}}{12}\left(\frac{1+2^{1+p}}{3(1+p)}\right)^{\frac{1}{p}}\left(\left(\frac{3\left|\psi^{\prime}\left(\lambda_{1}\right)\right|^{q}+\left|\psi^{\prime}\left(\lambda_{2}\right)\right|^{q}}{4}\right)^{\frac{1}{q}}+\left(\frac{\left|\psi^{\prime}\left(\lambda_{1}\right)\right|^{q}+3\left|\psi^{\prime}\left(\lambda_{2}\right)\right|^{q}}{4}\right)^{\frac{1}{q}}\right) .
\end{aligned}
$$

Moreover, if we choose $w(u)=\frac{1}{\lambda_{2}-\lambda_{1}}$, we have Theorem 1.7 from [11].

Theorem 2.10. Let $\psi:\left[\lambda_{1}, \lambda_{2}\right] \rightarrow R$ be a differentiable function on $\left(\lambda_{1}, \lambda_{2}\right)$ such that $\psi^{\prime} \in$ $\mathcal{L}\left[\lambda_{1}, \lambda_{2}\right]$ with $0 \leqslant \lambda_{1}<\lambda_{2}$, and let $w:\left[\lambda_{1}, \lambda_{2}\right] \rightarrow R$ be continuous and symmetric function with respect to $\frac{\lambda_{1}+\lambda_{2}}{2}$. If $\left|\psi^{\prime}\right|^{\mathrm{q}}$ is s-convex in the second sense for some fixed $\mathrm{s} \in(0,1]$ and $\mathrm{q} \geqslant 1$, then

$$
\begin{aligned}
& \left|\frac{1}{6}\left(\psi\left(\lambda_{1}\right)+4 \psi\left(\frac{\lambda_{1}+\lambda_{2}}{2}\right)+\psi\left(\lambda_{2}\right)\right)\left(\int_{\lambda_{1}}^{\lambda_{2}} w(u) d u\right)-\int_{\lambda_{1}}^{\lambda_{2}} w(u) \psi(u) d u\right| \\
\leqslant & \frac{3\left(\lambda_{2}-\lambda_{1}\right)^{2}}{4}\|w\|_{\left[\lambda_{1}, \lambda_{2}\right], \infty}\left(\frac{5}{54}\right)^{1-\frac{1}{q}}\left(\frac{1}{(1+s)(2+s)}\right)^{\frac{1}{q}} \\
& \times\left(\left(\left(\frac{s-1}{9}+\left(\frac{2}{3}\right)^{3+s}\right)\left|\psi^{\prime}\left(\lambda_{1}\right)\right|^{q}+\left(\frac{1+2 s}{9}+2\left(\frac{1}{3}\right)^{3+s}\right)\left|\psi^{\prime}\left(\frac{\lambda_{1}+\lambda_{2}}{2}\right)\right|^{q}\right)^{\frac{1}{q}}\right. \\
& \left.+\left(\left(\frac{1+2 s}{9}+2\left(\frac{1}{3}\right)^{3+s}\right)\left|\psi^{\prime}\left(\frac{\lambda_{1}+\lambda_{2}}{2}\right)\right|^{q}+\left(\frac{s-1}{9}+\left(\frac{2}{3}\right)^{3+s}\right)\left|\psi^{\prime}\left(\lambda_{2}\right)\right|^{q}\right)^{\frac{1}{q}}\right) .
\end{aligned}
$$

Proof. From Lemma 2.1, properties of modulus, power mean inequality, s-convexity of $\left|\psi^{\prime}\right|^{\mathrm{q}},(2.8)$ and (2.9), we have

$$
\begin{aligned}
& \left|\frac{1}{6}\left(\psi\left(\lambda_{1}\right)+4 \psi\left(\frac{\lambda_{1}+\lambda_{2}}{2}\right)+\psi\left(\lambda_{2}\right)\right)\left(\int_{\lambda_{1}}^{\lambda_{2}} w(u) d u\right)-\int_{\lambda_{1}}^{\lambda_{2}} w(u) \psi(u) d u\right| \\
& \leqslant \frac{3\left(\lambda_{2}-\lambda_{1}\right)^{2}}{4}\left(\int_{0}^{1}\left|p_{1}(x)\right| d x\right)^{1-\frac{1}{q}}\left(\int_{0}^{1}\left|p_{1}(x)\right|\left|\psi^{\prime}\left(x \lambda_{1}+(1-\chi) \frac{\lambda_{1}+\lambda_{2}}{2}\right)\right|^{q} d x\right)^{\frac{1}{q}} \\
& +\frac{3\left(\lambda_{2}-\lambda_{1}\right)^{2}}{4}\left(\int_{0}^{1}\left|p_{2}(x)\right| d x\right)^{1-\frac{1}{q}}\left(\int_{0}^{1}\left|p_{2}(x)\right|\left|\psi^{\prime}\left(\chi \frac{\lambda_{1}+\lambda_{2}}{2}+(1-x) \lambda_{2}\right)\right|^{q} d x\right)^{\frac{1}{q}} \\
& \leqslant \frac{3\left(\lambda_{2}-\lambda_{1}\right)^{2}}{4}\|w\|_{\left[\lambda_{1}, \lambda_{2}\right], \infty}\left(\int_{0}^{1}\left|\frac{2}{9}-\frac{1}{3} \chi\right| d \chi\right)^{1-\frac{1}{q}}
\end{aligned}
$$




$$
\begin{aligned}
& \times\left(\int_{0}^{1}\left|\frac{2}{9}-\frac{1}{3} \chi\right|\left(\chi^{\mathrm{s}}\left|\psi^{\prime}\left(\lambda_{1}\right)\right|^{\mathrm{q}}+(1-\chi)^{\mathrm{s}}\left|\psi^{\prime}\left(\frac{\lambda_{1}+\lambda_{2}}{2}\right)\right|^{\mathrm{q}}\right) \mathrm{d} \chi\right)^{\frac{1}{q}} \\
& +\frac{3\left(\lambda_{2}-\lambda_{1}\right)^{2}}{4}\|w\|_{\left[\lambda_{1}, \lambda_{2}\right], \infty}\left(\int_{0}^{1}\left|\frac{1}{9}-\frac{1}{3} \chi\right| \mathrm{d} \chi\right)^{1-\frac{1}{q}} \\
& \times\left(\int_{0}^{1}\left|\frac{1}{9}-\frac{1}{3} \chi\right|\left(\chi^{\mathrm{s}}\left|\psi^{\prime}\left(\frac{\lambda_{1}+\lambda_{2}}{2}\right)\right|^{\mathrm{q}}+(1-\chi)^{\mathrm{s}}\left|\psi^{\prime}\left(\lambda_{2}\right)\right|^{\mathrm{q}}\right) \mathrm{d} \chi\right)^{\frac{1}{q}} \\
& =\frac{3\left(\lambda_{2}-\lambda_{1}\right)^{2}}{4}\|w\|_{\left[\lambda_{1}, \lambda_{2}\right], \infty}\left(\int_{0}^{1}\left|\frac{2}{9}-\frac{1}{3} \chi\right| d \chi\right)^{1-\frac{1}{q}} \\
& \times\left(\left|\psi^{\prime}\left(\lambda_{1}\right)\right|^{\mathrm{q}} \int_{0}^{1}\left|\frac{2}{9}-\frac{1}{3} \chi\right| \chi^{\mathrm{s}} \mathrm{d} \chi+\left|\psi^{\prime}\left(\frac{\lambda_{1}+\lambda_{2}}{2}\right)\right|^{\mathrm{q}} \int_{0}^{1}\left|\frac{2}{9}-\frac{1}{3} \chi\right|(1-\chi)^{\mathrm{s}} \mathrm{d} \chi\right)^{\frac{1}{q}} \\
& +\frac{3\left(\lambda_{2}-\lambda_{1}\right)^{2}}{4}\|w\|_{\left[\lambda_{1}, \lambda_{2}\right], \infty}\left(\int_{0}^{1}\left|\frac{1}{9}-\frac{1}{3} \chi\right| d \chi\right)^{1-\frac{1}{q}} \\
& \times\left(\left|\psi^{\prime}\left(\frac{\lambda_{1}+\lambda_{2}}{2}\right)\right|^{\mathrm{q}} \int_{0}^{1}\left|\frac{1}{9}-\frac{1}{3} \chi\right| \chi^{\mathrm{s}} \mathrm{d} \chi+\left|\psi^{\prime}\left(\lambda_{2}\right)\right|^{\mathrm{q}} \int_{0}^{1}\left|\frac{1}{9}-\frac{1}{3} \chi\right|(1-\chi)^{s} \mathrm{~d} \chi\right)^{\frac{1}{q}} \\
& =\frac{3\left(\lambda_{2}-\lambda_{1}\right)^{2}}{4}\|w\|_{\left[\lambda_{1}, \lambda_{2}\right], \infty}\left(\frac{5}{54}\right)^{1-\frac{1}{q}}\left(\frac{1}{(1+s)(2+s)}\right)^{\frac{1}{q}} \\
& \times\left(\left(\left(\frac{s-1}{9}+\left(\frac{2}{3}\right)^{3+s}\right)\left|\psi^{\prime}\left(\lambda_{1}\right)\right|^{q}+\left(\frac{1+2 s}{9}+2\left(\frac{1}{3}\right)^{3+s}\right)\left|\psi^{\prime}\left(\frac{\lambda_{1}+\lambda_{2}}{2}\right)\right|^{q}\right)^{\frac{1}{q}}\right. \\
& \left.+\left(\left(\frac{1+2 s}{9}+2\left(\frac{1}{3}\right)^{3+s}\right)\left|\psi^{\prime}\left(\frac{\lambda_{1}+\lambda_{2}}{2}\right)\right|^{q}+\left(\frac{s-1}{9}+\left(\frac{2}{3}\right)^{3+s}\right)\left|\psi^{\prime}\left(\lambda_{2}\right)\right|^{q}\right)^{\frac{1}{q}}\right) \text {. }
\end{aligned}
$$

The proof is completed. 
Corollary 2.11. In Theorem 2.10, if we take $w(\mathrm{u})=\frac{1}{\lambda_{2}-\lambda_{1}}$, we get

$$
\begin{aligned}
& \left|\frac{1}{6}\left(\psi\left(\lambda_{1}\right)+4 \psi\left(\frac{\lambda_{1}+\lambda_{2}}{2}\right)+\psi\left(\lambda_{2}\right)\right)-\frac{1}{\lambda_{2}-\lambda_{1}} \int_{\lambda_{1}}^{\lambda_{2}} \psi(u) d u\right| \\
\leqslant & \frac{3\left(\lambda_{2}-\lambda_{1}\right)}{4}\left(\frac{5}{54}\right)^{1-\frac{1}{q}}\left(\frac{1}{(1+s)(2+s)}\right)^{\frac{1}{q}} \\
& \times\left(\left(\left(\frac{s-1}{9}+\left(\frac{2}{3}\right)^{3+s}\right)\left|\psi^{\prime}\left(\lambda_{1}\right)\right|^{q}+\left(\frac{1+2 s}{9}+2\left(\frac{1}{3}\right)^{3+s}\right)\left|\psi^{\prime}\left(\frac{\lambda_{1}+\lambda_{2}}{2}\right)\right|^{q}\right)^{\frac{1}{q}}\right. \\
& \left.+\left(\left(\frac{1+2 s}{9}+2\left(\frac{1}{3}\right)^{3+s}\right)\left|\psi^{\prime}\left(\frac{\lambda_{1}+\lambda_{2}}{2}\right)\right|^{q}+\left(\frac{s-1}{9}+\left(\frac{2}{3}\right)^{3+s}\right)\left|\psi^{\prime}\left(\lambda_{2}\right)\right|^{q}\right)^{\frac{1}{q}}\right) .
\end{aligned}
$$

Corollary 2.12. In Theorem 2.10, if we take $s=1$, we obtain

$$
\begin{aligned}
& \left|\frac{1}{6}\left(\psi\left(\lambda_{1}\right)+4 \psi\left(\frac{\lambda_{1}+\lambda_{2}}{2}\right)+\psi\left(\lambda_{2}\right)\right)\left(\int_{\lambda_{1}}^{\lambda_{2}} w(u) d u\right)-\int_{\lambda_{1}}^{\lambda_{2}} w(u) \psi(u) d u\right| \\
\leqslant & \frac{5\left(\lambda_{2}-\lambda_{1}\right)^{2}}{72}\|w\|_{\left[\lambda_{1}, \lambda_{2}\right], \infty} \\
& \times\left(\left(\frac{16\left|\psi^{\prime}\left(\lambda_{1}\right)\right|^{q}+29\left|\psi^{\prime}\left(\frac{\lambda_{1}+\lambda_{2}}{2}\right)\right|^{q}}{45}\right)^{\frac{1}{q}}+\left(\frac{29\left|\psi^{\prime}\left(\frac{\lambda_{1}+\lambda_{2}}{2}\right)\right|^{q}+16\left|\psi^{\prime}\left(\lambda_{2}\right)\right|^{q}}{45}\right)^{\frac{1}{q}}\right) .
\end{aligned}
$$

Moreover, if we choose $w(\mathrm{u})=\frac{1}{\lambda_{2}-\lambda_{1}}$, we have

$$
\begin{aligned}
& \left|\frac{1}{6}\left(\psi\left(\lambda_{1}\right)+4 \psi\left(\frac{\lambda_{1}+\lambda_{2}}{2}\right)+\psi\left(\lambda_{2}\right)\right)-\frac{1}{\lambda_{2}-\lambda_{1}} \int_{\lambda_{1}}^{\lambda_{2}} \psi(u) d u\right| \\
& \leqslant \frac{5\left(\lambda_{2}-\lambda_{1}\right)}{72}\left(\left(\frac{16\left|\psi^{\prime}\left(\lambda_{1}\right)\right|^{q}+29\left|\psi^{\prime}\left(\frac{\lambda_{1}+\lambda_{2}}{2}\right)\right|^{q}}{45}\right)^{\frac{1}{q}}+\left(\frac{29\left|\psi^{\prime}\left(\frac{\lambda_{1}+\lambda_{2}}{2}\right)\right|^{q}+16\left|\psi^{\prime}\left(\lambda_{2}\right)\right|^{q}}{45}\right) .\right.
\end{aligned}
$$

Corollary 2.13. In Corollary 2.12, using the convexity of $\left|\psi^{\prime}\right|^{q}$, we get

$$
\begin{aligned}
& \left|\frac{1}{6}\left(\psi\left(\lambda_{1}\right)+4 \psi\left(\frac{\lambda_{1}+\lambda_{2}}{2}\right)+\psi\left(\lambda_{2}\right)\right)\left(\int_{\lambda_{1}}^{\lambda_{2}} w(u) d u\right)-\int_{\lambda_{1}}^{\lambda_{2}} w(u) \psi(u) d u\right| \\
\leqslant & \frac{5\left(\lambda_{2}-\lambda_{1}\right)^{2}}{72}\|w\|_{\left[\lambda_{1}, \lambda_{2}\right], \infty} \\
& \times\left(\left(\frac{61\left|\psi^{\prime}\left(\lambda_{1}\right)\right|^{q}+29\left|\psi^{\prime}\left(\lambda_{2}\right)\right|^{q}}{90}\right)^{\frac{1}{q}}+\left(\frac{29\left|\psi^{\prime}\left(\lambda_{1}\right)\right|^{q}+61\left|\psi^{\prime}\left(\lambda_{2}\right)\right|^{q}}{90}\right)^{\frac{1}{q}}\right) .
\end{aligned}
$$


Moreover, if we choose $w(u)=\frac{1}{\lambda_{2}-\lambda_{1}}$, we obtain Theorem 1.8 from [11].

\section{Applications}

\subsection{Special means}

We shall consider the following special means for different positive real numbers $\lambda_{1}$ and $\lambda_{2}$.

- Arithmetic mean: $\mathcal{A}\left(\lambda_{1}, \lambda_{2}\right)=\frac{\lambda_{1}+\lambda_{2}}{2}$.

- p-Logarithmic mean: $\mathcal{L}_{p}\left(\lambda_{1}, \lambda_{2}\right)=\left(\frac{\lambda_{2}^{p+1}-\lambda_{1}^{p+1}}{(p+1)\left(\lambda_{2}-\lambda_{1}\right)}\right)^{\frac{1}{p}}, p \in \mathbb{Z} \backslash\{0,-1\}$.

Proposition 3.1. Let $\lambda_{1}, \lambda_{2} \in \mathrm{R}$ and $0<\lambda_{1}<\lambda_{2}$. Then

$$
\left|\mathcal{A}\left(\lambda_{1}^{3}, \lambda_{2}^{3}\right)+2 \mathcal{A}^{3}\left(\lambda_{1}, \lambda_{2}\right)-3 \mathcal{L}_{3}^{3}\left(\lambda_{1}, \lambda_{2}\right)\right| \leqslant \frac{\lambda_{2}-\lambda_{1}}{36}\left(16 \mathcal{A}\left(\lambda_{1}^{2}, \lambda_{2}^{2}\right)+29 \mathcal{A}^{2}\left(\lambda_{1}, \lambda_{2}\right)\right) .
$$

Proof. The assertion follows from Theorem 2.2 with $w(u)=\frac{1}{\lambda_{2}-\lambda_{1}}$ and $s=1$, applied to the function $\psi(u)=u^{3}$.

Proposition 3.2. Let $\lambda_{1}, \lambda_{2} \in \mathrm{R}$ and $0<\lambda_{1}<\lambda_{2}$. Then for $\mathrm{q} \geqslant 1$, we have

$$
\begin{aligned}
& \left|\mathcal{A}\left(\lambda_{1}^{\frac{1+2 q}{2 q}}, \lambda_{2}^{\frac{1+2 q}{2 q}}\right)+2 \mathcal{A}^{\frac{1+2 q}{2 q}}\left(\lambda_{1}, \lambda_{2}\right)-3 \mathcal{L}_{\frac{1+2 q}{2 q}}^{\frac{1+2 q}{2 q}}\left(\lambda_{1}, \lambda_{2}\right)\right| \\
\leqslant & \frac{5\left(\lambda_{2}-\lambda_{1}\right)}{24} \frac{1+2 q}{2 q}\left(\left(\frac{4(16 \sqrt{6}-9) \sqrt{\lambda_{1}}+16(9+\sqrt{3}) \sqrt{\frac{\lambda_{1}+\lambda_{2}}{2}}}{225}\right)^{\frac{1}{q}}\right. \\
& \left.+\left(\frac{16(9+\sqrt{3}) \sqrt{\frac{\lambda_{1}+\lambda_{2}}{2}}+4(16 \sqrt{6}-9) \sqrt{\lambda_{2}}}{225}\right)^{\frac{1}{q}}\right)
\end{aligned}
$$

Proof. The assertion follows from Corollary 2.11, applied to the function $\psi(u)=\frac{2 q}{1+2 q} u^{\frac{1+2 q}{2 q}}$ with $q \geqslant 1$, which clearly $\left|\psi^{\prime}(u)\right|^{q}$ is $\frac{1}{2}$-convex.

3.2. Weighted Simpson quadrature formula

Let $\mathcal{P}$ be the partition of the points $\lambda_{1}=x_{0}<x_{1}<\ldots<x_{n}=\lambda_{2}$ of the interval $\left[\lambda_{1}, \lambda_{2}\right]$ and consider the quadrature formula

$$
\int_{\lambda_{1}}^{\lambda_{2}} w(u) \psi(u) d u=\mathcal{S}_{w}(\psi, \mathcal{P})+\mathcal{R}_{w}(\psi, \mathcal{P})
$$

where

$$
\mathcal{S}_{w}(\psi, \mathcal{P}):=\frac{1}{6} \sum_{i=0}^{n-1}\left[\psi\left(x_{i}\right)+4 \psi\left(\frac{x_{i}+x_{i+1}}{2}\right)+\psi\left(x_{i+1}\right)\right]\left(\int_{x_{i}}^{x_{i+1}} w(u) d u\right),
$$


is the weighted Simpson version and $\mathcal{R}_{w}(\psi, \mathcal{P})$ denotes the associated approximation error. The following results are given to illustrative the implementation of above weighted Simpson quadrature formula.

Proposition 3.3. Let $\mathrm{n} \in \mathbb{N}$ and $\psi:\left[\lambda_{1}, \lambda_{2}\right] \rightarrow \mathrm{R}$ be a differentiable function on $\left(\lambda_{1}, \lambda_{2}\right)$ such that $\psi^{\prime} \in \mathcal{L}\left[\lambda_{1}, \lambda_{2}\right]$ with $0 \leqslant \lambda_{1}<\lambda_{2}$, and let $w:\left[\lambda_{1}, \lambda_{2}\right] \rightarrow R$ be continuous and symmetric function with respect to $\frac{\lambda_{1}+\lambda_{2}}{2}$. If $\left|\psi^{\prime}\right|$ is s-convex in the second sense for some fixed $s \in(0,1]$, then

$$
\begin{aligned}
& \left|\mathcal{R}_{w}(\psi, \mathcal{P})\right| \leqslant \frac{3}{4(1+s)(2+s)}\|w\|_{\left[\lambda_{1}, \lambda_{2}\right], \infty} \\
& \times \sum_{i=0}^{n-1}\left(x_{i+1}-x_{i}\right)^{2}\left(\left(\frac{s-1}{9}+\left(\frac{2}{3}\right)^{3+s}\right)\left|\psi^{\prime}\left(x_{i}\right)\right|\right. \\
& \left.\quad+2\left(\frac{1+2 s}{9}+2\left(\frac{1}{3}\right)^{3+s}\right)\left|\psi^{\prime}\left(\frac{x_{i}+x_{i+1}}{2}\right)\right|+\left(\frac{s-1}{9}+\left(\frac{2}{3}\right)^{3+s}\right)\left|\psi^{\prime}\left(x_{i+1}\right)\right|\right) .
\end{aligned}
$$

Proof. Applying Theorem 2.2 on the subintervals $\left[x_{i}, x_{i+1}\right](i=0, \ldots, n-1)$ of the partition $\mathcal{P}$, we get

$$
\begin{aligned}
& \quad\left|\int_{x_{i}}^{x_{i+1}} w(u) \psi(u) d u-\frac{1}{6}\left[\psi\left(x_{i}\right)+4 \psi\left(\frac{x_{i}+x_{i+1}}{2}\right)+\psi\left(x_{i+1}\right)\right]\left(\int_{x_{i}}^{x_{i+1}} w(u) d u\right)\right| \\
& \leqslant \frac{3}{4(1+s)(2+s)}\|w\|_{\left[x_{i}, x_{i+1}\right], \infty} \\
& \times\left(x_{i+1}-x_{i}\right)^{2}\left(\left(\frac{s-1}{9}+\left(\frac{2}{3}\right)^{3+s}\right)\left|\psi^{\prime}\left(x_{i}\right)\right|\right. \\
& \left.\quad+2\left(\frac{1+2 s}{9}+2\left(\frac{1}{3}\right)^{3+s}\right)\left|\psi^{\prime}\left(\frac{x_{i}+x_{i+1}}{2}\right)\right|+\left(\frac{s-1}{9}+\left(\frac{2}{3}\right)^{3+s}\right)\left|\psi^{\prime}\left(x_{i+1}\right)\right|\right) .
\end{aligned}
$$

Hence, summing above inequality for all $i=0, \ldots, n-1$ and using property of modulus, we have the desired result.

Proposition 3.4. Let $\mathrm{n} \in \mathbb{N}$ and $\psi:\left[\lambda_{1}, \lambda_{2}\right] \rightarrow R$ be a differentiable function on $\left(\lambda_{1}, \lambda_{2}\right)$ such that $\psi^{\prime} \in \mathcal{L}\left[\lambda_{1}, \lambda_{2}\right]$ with $0 \leqslant \lambda_{1}<\lambda_{2}$, and let $w:\left[\lambda_{1}, \lambda_{2}\right] \rightarrow R$ be continuous and symmetric function with respect to $\frac{\lambda_{1}+\lambda_{2}}{2}$. If $\left|\psi^{\prime}\right|^{q}$ is s-convex in the second sense for some fixed $s \in(0,1]$, where $\mathrm{q}>1$ with $\frac{1}{\mathrm{p}}+\frac{1}{\mathrm{q}}=1$, then

$$
\begin{aligned}
& \left|\mathcal{R}_{w}(\psi, \mathcal{P})\right| \leqslant \frac{1}{12}\|w\|_{\left[\lambda_{1}, \lambda_{2}\right], \infty}\left(\frac{1+2^{1+p}}{3(1+p)}\right)^{\frac{1}{p}}\left(\frac{1}{1+s}\right)^{\frac{1}{q}} \\
& \times \sum_{i=0}^{n-1}\left(x_{i+1}-x_{i}\right)^{2}\left(\left(\left|\psi^{\prime}\left(x_{i}\right)\right|^{q}+\left|\psi^{\prime}\left(\frac{x_{i}+x_{i+1}}{2}\right)\right|^{q}\right)^{\frac{1}{q}}+\left(\left|\psi^{\prime}\left(\frac{x_{i}+x_{i+1}}{2}\right)\right|^{q}+\left|\psi^{\prime}\left(x_{i+1}\right)\right|^{q}\right)^{\frac{1}{q}}\right) .
\end{aligned}
$$

Proof. Applying Theorem 2.6 using the same idea as in Proposition 3.3, we get the desired result. 
Proposition 3.5. Let $\mathrm{n} \in \mathbb{N}$ and $\psi:\left[\lambda_{1}, \lambda_{2}\right] \rightarrow \mathrm{R}$ be a differentiable function on $\left(\lambda_{1}, \lambda_{2}\right)$ such that $\psi^{\prime} \in \mathcal{L}\left[\lambda_{1}, \lambda_{2}\right]$ with $0 \leqslant \lambda_{1}<\lambda_{2}$, and let $w:\left[\lambda_{1}, \lambda_{2}\right] \rightarrow \mathrm{R}$ be continuous and symmetric function with respect to $\frac{\lambda_{1}+\lambda_{2}}{2}$. If $\left|\psi^{\prime}\right|^{\mathrm{q}}$ is s-convex in the second sense for some fixed $s \in(0,1]$ and $\mathrm{q} \geqslant 1$, then

$$
\begin{aligned}
& \left|\mathcal{R}_{w}(\psi, \mathcal{P})\right| \leqslant \frac{3}{4}\|w\|_{\left[\lambda_{1}, \lambda_{2}\right], \infty}\left(\frac{5}{54}\right)^{1-\frac{1}{q}}\left(\frac{1}{(1+s)(2+s)}\right)^{\frac{1}{q}} \\
& \times \sum_{i=0}^{n-1}\left(x_{i+1}-x_{i}\right)^{2}\left(\left(\left(\frac{s-1}{9}+\left(\frac{2}{3}\right)^{3+s}\right)\left|\psi^{\prime}\left(x_{i}\right)\right|^{q}+\left(\frac{1+2 s}{9}+2\left(\frac{1}{3}\right)^{3+s}\right)\left|\psi^{\prime}\left(\frac{x_{i}+x_{i+1}}{2}\right)\right|^{q}\right)^{\frac{1}{q}}\right. \\
& \left.+\left(\left(\frac{1+2 s}{9}+2\left(\frac{1}{3}\right)^{3+s}\right)\left|\psi^{\prime}\left(\frac{x_{i}+x_{i+1}}{2}\right)\right|^{q}+\left(\frac{s-1}{9}+\left(\frac{2}{3}\right)^{3+s}\right)\left|\psi^{\prime}\left(x_{i+1}\right)\right|^{q}\right)^{\frac{1}{q}}\right) .
\end{aligned}
$$

Proof. Applying Theorem 2.10 using the same idea as in Proposition 3.3, we obtain the required result.

\section{Conclusion}

The main results and future research of the article can be summarized as follows:

- A new identity regarding Simpson type is established.

- New weighted Simpson type inequalities for s-convex functions in the second sense using above identity are deduced.

- Various special cases have been studied in details.

- Some applications to special means are given.

- Some results are obtained to illustrative the implementation of weighted Simpson quadrature formula.

- We hope that our results can be applied to obtain several new results in different areas of pure and applied sciences.

\section{References}

[1] Pečarić JE, Proschan F and Tong YL (1992). Convex functions, partial orderings, and statistical applications. Math. Sci. Eng. 187.

[2] Breckner WW (1978). Stetigkeitsaussagen für eine Klasse verallgemeinerter konvexer Funktionen in topologischen linearen Räumen. Publ. Inst. Math. (Beograd) (N.S.) 23(37): 13-20.

[3] Awan MU, Noor MA, Mihai MV, Noor KI and Khan AG (2017). Some new bounds for Simpson's rule involving special functions via harmonic h-convexity. J. Nonlinear Sci. Appl. 10(4): 1755-1766.

[4] Chiheb T, Boumaza N and Meftah B (2020). Some new Simpson-like type inequalities via prequasi-invexity. Transylv. J. Math. Mech. 12(1): 1-10.

[5] Dragomir SS, Agarwal RP and Cerone P (2000). On Simpson's inequality and applications. J. Inequal. Appl. 5: 533-579.

[6] Kashuri A, Mohammed PO, Abdeljawad T, Hamasalh F and Chu YM (2020). New Simpson type integral inequalities for s-convex functions and their applications. Math. Probl. Eng. 2020 Article ID 8871988: 1-12. 
[7] Noor MA, Noor KI and Awan MU (2018). Simpson-type inequalities for geometrically relative convex functions. Ukrainian Math. J. 70(7): 1145-1154.

[8] Sarikaya MZ, Budak H and Erden S (2019). On new inequalities of Simpson's type for generalized convex functions. Korean J. Math. 27(2): 279-295.

[9] Set E, Özdemir ME and Sarikaya MZ (2012). On new inequalities of Simpson's type for quasi-convex functions with applications. Tamkang J. Math. 43(3): 357-364.

[10] Alomari M, Darus M and Dragomir SS (2009). New inequalities of Simpson's type for s-convex functions with applications. RGMIA Res. Rep. Coll. 12(4) Article 9.

[11] Sarikaya MZ, Set E and Özdemir ME (2010). On new inequalities of Simpson's type for convex functions. RGMIA Res. Rep. Coll. 13(2) Article 2.

[12] Sarikaya MZ, Set E and Özdemir ME (2010). On new inequalities of Simpson's type for s-convex functions. Comput. Math. Appl. 60(8): 2191-2199.

[13] Matłoka M (2017). Weighted Simpson type inequalities for h-convex functions. J. Nonlinear Sci. Appl. 10(11): 5770-5780.

[14] Abdeljawad T, Rashid S, Hammouch Z, Işcan I and Chu YM (2020). Some new Simpson-type inequalities for generalized p-convex function on fractal sets with applications. Adv. Difference Equ. 2020: 1. https: //doi.org/10.1186/s13662-020-02955-9

[15] Rashid S, Hammouch Z, Baleanu D and Chu YM (2020). New generalizations in the sense of the weighted non-singular fractional integral operator. Fractals 28(8): 11. https://doi.org/10.1142/ S0218348X20400034

[16] Rahman G, Nisar KS, Ghaffar A and Qi F (2020). Some inequalities of the Grüss type for conformable k-fractional integral operators. Rev. R. Acad. Cienc. Exactas Fìs. Nat., Ser. A Mat., RACSAM 114: 9. https://doi.org/10.1007/s13398-019-00731-3

[17] Rahman G, Abdeljawad T, Khan A and Nisar KS (2019). Some fractional proportional integral inequalities. J. Inequal. Appl. 2019: 244. https://doi.org/10.1186/s13660-019-2199-z

[18] Rahman G, Abdeljawad T, Jarad F, Khan A and Nisar KS (2019). Certain inequalities via generalized proportional Hadamard fractional integral operators. Adv. Difference Equ. 2019: 454. https://doi.org/ 10.1186/s13662-019-2381-0

[19] Rahman G, Khan A, Abdeljawad T and Nisar KS (2019). The Minkowski inequalities via generalized proportional fractional integral operators. Adv. Difference Equ. 2019: 287. https://doi.org/10.1186/ s13662-019-2229-7

[20] Nisar KS, Rahman G and Mehrez K (2019). Chebyshev type inequalities via generalized fractional conformable integrals. J. Inequal. Appl. 2019: 245. https://doi.org/10.1186/s13660-019-2197-1

[21] Nisar KS, Tassaddiq A, Rahman G and Khan A (2019). Some inequalities via fractional conformable integral operators. J. Inequal. Appl. 2019: 217. https://doi.org/10.1186/s13660-019-2170-z

[22] Baleanu D, Mohammed PO, Vivas-Cortez MJ and Rangel-Oliveros Y (2020). Some modifications in conformable fractional integral inequalities. Adv. Difference Equ. 2020: 374. https://doi.org/10.1186/ s13662-020-02837-0

[23] Abdeljawad T, Mohammed PO and Kashuri A (2020). New modified conformable fractional integral inequalities of Hermite-Hadamard type with applications. J. Funct. Spaces 2020 Article ID 4352357. https: //doi.org/10.1155/2020/4352357

[24] Mohammed PO (2016). Some integral inequalities of fractional quantum type. Malaya J. Mat. 4(1): $93-99$.

[25] Mohammed PO and Abdeljawad T (2020). Integral inequalities for a fractional operator of a function with respect to another function with nonsingular kernel. Adv. Difference Equ. 2020: 363. https://doi.org/ 10.1186/s13662-020-02825-4

[26] Mohammed PO and Brevik I (2020). A new version of the Hermite-Hadamard inequality for RiemannLiouville fractional integrals. Symmetry 12(4): 610. https ://doi .org/10.3390/sym12040610

[27] Mohammed PO and Sarikaya MZ (2020). On generalized fractional integral inequalities for twice differentiable convex functions. J. Comput. Appl. Math. 372 Article ID 112740. https://doi.org/10.1016/j . cam.2020.112740

[28] Baleanu D, Kashuri A, Mohammed PO and Meftah B (2021). General Raina fractional integral inequalities on coordinates of convex functions. Adv. Difference Equ. 2021: 82. https://doi.org/10.1186/ s13662-021-03241-y

[29] Alqudah MA, Kashuri A, Mohammed PO, Raees M and Abdeljawad T (2021). On modified convex interval valued functions and related inclusions via the interval valued generalized fractional integrals in extended interval space. AIMS Math. 6(5): 4638-4663. 
[30] Mohammed PO, Abdeljawad T, Baleanu D, Kashuri A, Hamasalh F and Agarwal P (2020). New fractional inequalities of Hermite-Hadamard type involving the incomplete gamma functions. J. Inequal. Appl. 2020: 263. https://doi.org/10.1186/s13660-020-02538-y 